\title{
Electromagnetic Scattering by Arbitrary Shaped Three-Dimensional Homogeneous Lossy Dielectric Objects
}

\author{
KORADA UMASHANKAR, SENIOR MEMBER, IFEE, ALLEN TAFLOVE, SENIOR MEMBER, IEEE, AND SADASIVA M. RAO
}

\begin{abstract}
The recent development and extension of the method of moments technique for analyzing electromagnetic scattering by arbitrary shaped three-dimensional homogeneous lossy dielectric objects is presented based on the combined field integral equations. The surfaces of the homogeneous three-dimensional arbitrary geometrical shapes are modeled using surface triangular patches, similar to the case of arbitrary shaped conducting objects. Further, the development and extensions required to treat efficiently three-dimensional lossy dielectric objects are reported. Numerical results and their comparisons are also presented for two canonical dielectric scatterers-a sphere and a finite circular cylinder.
\end{abstract}

\section{INTRODUCTION}

$\mathrm{T}$ HIS PAPER DEALS with the use of analytical and numerical methods to analyze electromagnetic scattering and corresponding radar cross section of three-dimensional arbitrary shaped homogeneous lossy dielectric objects. There is, in fact, a variety of frequency domain analytical and/or numerical methods applied for studying electromagnetic scattering by homogeneous dielectric objects. For those objects whose boundary surface just coincides with a given coordinate system, separation of variables [1] can be applied. For these cases, analytically exact solutions have been obtained only for simple scatterers, such as a sphere [2] and circular and elliptical cylinders [3]-[5]. For an object not much different from the sphere, namely a prolate spheroid, the perturbation technique [6] has been applied.

Also for objects which are arbitrary in shape, either the volume or the surface integral equation has been applied. The volume integral equation [7], [8] is principally based on relating the induced polarization currents to the corresponding total fields consisting of the scattering and incident fields. By associating an unknown polarization current coefficient either with a cubic cell or with a tetrahedral cell inside the scatterer [8], the operator form of the integral equation is converted into an equivalent matrix equation. To a limited extent, the recent

Manuscript received November 9, 1984. This work was sponsored by the Electromagnetic Sciences Division, the Rome Air Development Center, Hanscom Air Force Base, MA, under Contract F19628-82-C-0140 to IIT Research Institute, Chicago, IL.

$\mathrm{K}$. Umashankar is with the Communications Laboratory, Department of Electrical Engineering and Computer Science, University of Illinois at Chicago, IL 60680.

A. Taflove is with the Department of Electrical Engineering and Computer Science, Northwestern University, Evanston, IL 60201.

S. M. Rao is with the Department of Electrical Engineering, Rochester Institute of Technology, Rochester, NY 14623.

IEEE Log Number 8608070. advancements [9], [10] in the area of the fast Fourier transform and also iterative methods have extended the usefulness of the volume integral equation approaches. Employing the volume equivalence principle and method of moments, scattering results are reported for dielectric [3], [7] cylinders and also for plane slabs and biological tissue cylinders [11]. Some work is also reported in the area of the extended boundary condition approach [12] and also in the area of the unimoment method [13], [14] related to scattering by inhomogeneous objects. These approaches express fields in terms of integrals over surfaces separating one or more homogeneous regions surrounding a given scatterer [15], [16]. For detailed discussion on the various techniques and their limitations, the reader may refer to the recent technical report [17] by the authors of the present paper.

The surface integral equation approach is very well suited to analyzing homogeneous dielectric objects or to objects modeled by or made up of homogeneous layers [13], [18]. The usual procedure in this method is to set up coupled integral equations in terms of equivalent electric and magnetic currents on the surfaces of the homogeneous regions. For an object made up of a large number of layers, fields induced in any region are expressed in terms of the equivalent currents on the adjacent interfaces. An iterative procedure [18] has been utilized for solving currents on the outermost surface in terms of the currents on inner interfaces. Particularly, for the case of simple objects such as dielectric cylinders [5] and bodies of revolution [19], the surface coupled integral equations method has been extensively applied. But, when the surface of the scatterer takes on arbitrary shape, an efficient modeling of the surface geometry and also the surface electric and magnetic fields become complicated. A simple and an efficient modeling scheme is presented here and is the subject of discussion in this paper in the context of scattering by arbitrarily shaped objects [17], [20].

\section{Triangular Surface Patch Modeling}

General arbitrarily shaped scattering objects can be analyzed based on integral equations and the method of moments (MM) approach [17]. This method is suited for low-frequency scattering problems, but can be extended to bodies spanning approximately one to two wavelengths in three dimensions, Fig. 1. The following formulations of the MM have been found to be generally suited for certain scattering problems 


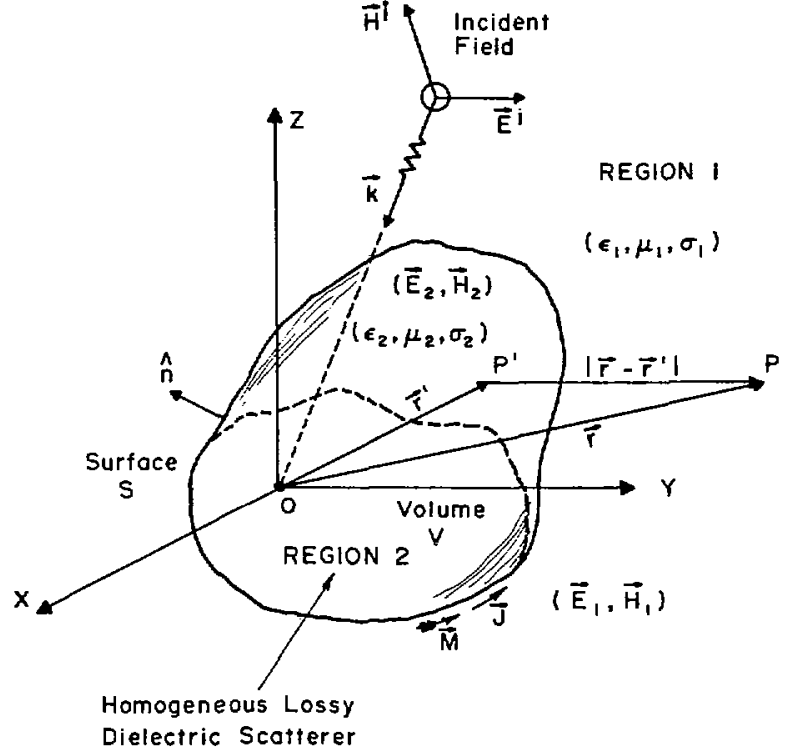

Fig. 1. Geometry of a homogeneous lossy dielectric scatterer in an isotropic free space medium.

based upon their geometry and material characteristics:

1) Conducting Scatterers (homogeneous, isotropic)

a) Electric field integration formulation (EFIE) for closed and open bodies

b) Magnetic field integral equation formulation (MFIE) for closed bodies

2) Dielectric Scatterers (homogeneous, isotropic)

Combined field integral equation formulation (CFIE)

3) Anisotropic Scatterers (homogeneous)

Combined field integral equation formulation (CFIE) modified for material characteristics.

Recent work has concentrated on case 1 , especially in the development of the triangular patch model for arbitrary scatterers [20]. Recent further work is reported [17], using the combined field formulation for cases 2 and 3 ; only case 2 is discussed in this paper. There are several approaches by which one can efficiently model a given arbitrary shaped surface. For planar surface, one can conveniently use either rectangular or square patches [19]. Difficulties arise in case of nonplanar surfaces and even in case of planar surfaces with irregular boundary edges. An elaborate discussion on the triangular surface patch techniques, orientation of patches and their applicabilities to various types of integrodifferential equations is well discussed in [21]. Since the analysis of both EFIE and MFIE are already studied for conducting bodies [21], necessary analytical and numerical developments can be conveniently developed and extended [17] to surface patch model any arbitrary shaped dielectric objects, for any given excitation. Using MM to solve these equations, the equivalent electric and magnetic surface currents are expanded in terms of triangular surface patch currents; and tested on both sides with respect to the same surface patch basis functions to yield an efficient matrix expression. Once the surface equivalent currents are known, the near scattered fields, scattered far fields, and even penetrated fields are directly evaluated. To demonstrate validity, numerical results and their comparisons are presented for two canonical dielectric scatterers, namely a homogeneous dielectric sphere and a finite circular cylinder.

\section{Summary of Combined Field Integral Equations}

The detail derivation of the combined field integral equations can be found in [17] and [22], but for completeness and further numerical development only a summary of the CFIE equations is given below. Referring to Fig. 1, $S$ denotes the surface of a homogeneous, lossy dielectric scatterer having a volume $V$ contained in region 2 and bounded by the surface $S$. The scatterer is located in region 1 representing an isotropic, lossless free space medium. Let

$\left(\vec{E}_{1}^{s}, \vec{H}_{1}^{s}\right)$

$=$ scattered electric and magnetic fields in region 1

$\left(\vec{E}_{2}^{s}, \vec{H}_{2}^{s}\right)$

$=$ scattered electric and magnetic fields in region 2.

Then, referring to the electromagnetic equivalent principle, various scattered electric and magnetic fields in regions 1 and 2 are given by

$$
\begin{aligned}
& \vec{E}_{1}^{s}(\vec{r})=-j \omega \vec{A}_{1}(\vec{r})-\nabla V_{1}(\vec{r})-\frac{1}{\epsilon_{1}^{\prime}} \nabla \times \vec{F}_{1}(\vec{r}) \\
& \vec{H}_{1}^{s}(\vec{r})=-j \omega \vec{F}_{1}(\vec{r})-\nabla U_{1}(\vec{r})+\frac{1}{\mu_{1}} \nabla \times \vec{A}_{1}(\vec{r}),
\end{aligned}
$$

for $\vec{r}$ on or outside $S$

$$
\begin{aligned}
& \vec{E}_{2}^{s}(\vec{r})=j \omega \vec{A}_{2}(\vec{r})+\nabla V_{2}(\vec{r})+\frac{1}{\epsilon_{2}^{\prime}} \nabla \times \vec{F}_{2}(\vec{r}) \\
& \vec{H}_{2}^{s}(\vec{r})=j \omega \vec{F}_{2}(\vec{r})+\nabla U_{2}(\vec{r})-\frac{1}{\mu_{2}} \nabla \times \vec{A}_{2}(\vec{r}),
\end{aligned}
$$

$$
\text { for } \vec{r} \text { on or inside } S
$$

where the various vector potentials $\vec{A}_{i}$ and $\vec{F}_{i}$ and the scalar potentials $V_{i}$ and $U_{i}$, for $i=1,2$ are given by

$$
\begin{gathered}
\vec{A}_{i}(\vec{r})=\frac{\mu_{i}}{4 \pi} \iint_{S} \vec{J}\left(\vec{r}^{\prime}\right) G_{i}\left(\vec{r}, \vec{r}^{\prime}\right) d S\left(\vec{r}^{\prime}\right) \\
\vec{F}_{i}(\vec{r})=\frac{\epsilon_{i}^{\prime}}{4 \pi} \iint_{S} \vec{M}\left(\vec{r}^{\prime}\right) G_{i}\left(\vec{r}, \vec{r}^{\prime}\right) d S\left(\vec{r}^{\prime}\right) \\
V_{i}(\vec{r})=\frac{1}{4 \pi \epsilon_{i}^{\prime}} \iint_{S} \rho^{e}\left(\vec{r}^{\prime}\right) G_{i}\left(\vec{r}, \vec{r}^{\prime}\right) d S\left(\vec{r}^{\prime}\right) \\
U_{i}(\vec{r})=\frac{1}{4 \pi \mu_{i}} \iint_{S} \rho^{m}\left(\vec{r}^{\prime}\right) G_{i}\left(\vec{r}, \vec{r}^{\prime}\right) d S\left(\vec{r}^{\prime}\right) \\
\epsilon_{i}^{\prime}=\epsilon_{i}\left[1-j \frac{\sigma_{i}}{\omega \epsilon_{i}}\right]
\end{gathered}
$$


and

$$
\begin{aligned}
\rho^{e}\left(\vec{r}^{\prime}\right) & =\frac{-1}{j \omega}\left[\nabla_{S}^{\prime} \cdot \vec{J}\left(\vec{r}^{\prime}\right)\right] \\
\rho^{m}\left(\vec{r}^{\prime}\right) & =\frac{-1}{j \omega}\left[\nabla_{S}^{\prime} \cdot \vec{M}\left(\vec{r}^{\prime}\right)\right] .
\end{aligned}
$$

In obtaining the above expressions, $e^{j \omega t}$ time dependence is assumed for various field quantities and $\omega$ is the frequency in radians per second. The Green's function defined in (3a)-(3d) for $i=1,2$ is given by

$$
\begin{gathered}
G_{i}\left(\vec{r}, \vec{r}^{\prime}\right)=\frac{e^{-j k_{i} R}}{R} \\
R=\left|\vec{r}-\vec{r}^{\prime}\right|
\end{gathered}
$$

and the propagation constant is

$$
k_{i}=\left[\omega^{2} \mu_{i} \epsilon_{i}^{\prime}\right]^{1 / 2} \text {. }
$$

In (3a) and (3b), $\vec{J}$ is the equivalent electric current and $\vec{M}$ is the equivalent magnetic current on the surface of the dielectric scatterer. The equivalent electric and magnetic currents are, in fact, related to the surface total magnetic and electric fields tangential to the surface $S$ :

$$
\begin{aligned}
& \vec{J}\left(\vec{r}^{\prime}\right)=\hat{n} \times \vec{H}\left(\vec{r}^{\prime}\right) \\
& \vec{M}\left(\vec{r}^{\prime}\right)=\vec{E}\left(\vec{r}^{\prime}\right) \times \hat{n}, \quad \vec{r}^{\prime} \text { on the surface } S
\end{aligned}
$$

where $\hat{n}$ is an outward unit normal on $S$ shown in Fig. 1 . Further, in the above expressions, $\left(\epsilon_{1}, \mu_{1}, \sigma_{1}=0\right)$ and $\left(\epsilon_{2}, \mu_{2}\right.$, $\sigma_{2}$ ) are the permittivity, permeability, and conductivity for the regions 1 and 2.

On enforcing the boundary condition that the total tangential electric field and the total tangential magnetic field should be continuous across the surface of the arbitrary dielectric scatterer, the following combined field integral equations are obtained in terms of the unknown surface equivalent electric and magnetic currents:

$$
\begin{array}{r}
\left.\vec{E}^{i}(\vec{r})\right|_{\tan }=\left\{j \omega\left[\vec{A}_{1}(\vec{r})+\vec{A}_{2}(\vec{r})\right]+\left[\nabla V_{1}(\vec{r})+\nabla V_{2}(\vec{r})\right]\right. \\
\left.+\nabla \times\left[\frac{\vec{F}_{1}(\vec{r})}{\epsilon_{1}^{\prime}}+\frac{\vec{F}_{2}(\vec{r})}{\epsilon_{2}^{\prime}}\right]\right\}\left.\right|_{\tan } \\
\left.\vec{H}^{i}(\vec{r})\right|_{\tan }=\left\{j \omega\left[\vec{F}_{1}(\vec{r})+\vec{F}_{2}(\vec{r})\right]+\left[\nabla U_{1}(\vec{r})+\nabla U_{2}(\vec{r})\right]\right. \\
\left.-\nabla \times\left[\frac{\vec{A}_{1}(\vec{r})}{\mu_{1}}+\frac{\vec{A}_{2}(\vec{r})}{\mu_{2}}\right]\right\}\left.\right|_{\tan }, \quad \vec{r} \text { on surface } S
\end{array}
$$

where $\vec{E}^{i}$ and $\vec{H}^{i}$ are the incident electric and magnetic fields in the region 1 and the subscript "tan" refers to tangential component only. A detailed numerical approach is discussed in the following based on the method of moment technique to reduce the coupled integrodifferential equations (8a) and (8b) to the corresponding partitioned matrix equation for the unknown electric and magnetic currents on the surface of the scatterer. Especially to treat arbitrary shaped bodies, the surface $S$ of the dielectric scatterer should be efficiently modeled as proposed [21] by dividing the surface of the scatterer into a number of triangular shaped surface patches. An example is shown in Fig. 2 indicating how one can use triangles to patch model scatterer surfaces efficiently. Fig. 2 shows the case of a finite circular dielectric cylinder scatterer modeled in terms of triangular surface patches. In each of the triangular surface patch, the electric and magnetic currents are represented in terms of a known triangular basis function. The complete development of current expansion or basis functions for triangular surface patches suitable for homogeneous dielectric objects is discussed in detail in [17].

\section{Basis Functions and Current Representation}

Given a closed surface $S$ the surface is first approximated by a number of triangles, Fig. 2. Each triangle is defined by an appropriate set of faces, edges, and vertices. Fig. 3(a) shows two triangles $T_{n}^{+}$and $T_{n}^{-}$with the $n$th common edge. The electric and magnetic currents flow along radial direction $\hat{\rho}_{n}^{+}$ in triangle $T_{n}^{+}$and similarly flow along radial direction $\hat{\rho}_{n}^{-}$in triangle $T_{n}^{-}$. Referring to Fig. 3(b), if $l_{n}$ is the base length of common edge, then height lengths of the two triangles $T_{n}^{+}$and $T_{n}^{-}$are, respectively, given by $2 A_{n}^{+} / l_{n}$ and $2 A_{n}^{-} / l_{n}$, where $A_{n}^{ \pm}$represents the area of $T_{n}^{ \pm}$. Any point in triangles $T_{n}^{ \pm}$can be defined either with respect to global origin, $O$, or with respect to the triangle vertices $O_{n}^{ \pm}$. In Fig. 3, the superscripts plus and minus signs designation of the triangles is determined by choice of a positive current reference direction [21] for the $n$th edge, which is always assumed to be from $T_{n}^{+}$to $T_{n}^{-}$. Hence, a vector basis function associated with $n$th edge is

$$
\vec{f}_{n}(\vec{r})= \begin{cases}\frac{l_{n}}{2 A_{n}^{+}} \vec{\rho}_{n}^{+}, & \vec{r} \text { in } T_{n}^{+} \\ \frac{l_{n}}{2 A_{n}^{-}} \vec{\rho}_{n}^{-}, & \vec{r} \text { in } T_{n}^{-} \\ \overrightarrow{0}, & \text { otherwise. }\end{cases}
$$

The vector basis function stated in (9) is ideally suited for representing surface electric current $\vec{J}$ and the surface magnetic current $\vec{M}$ on the triangulated surface $S$ of the given dielectric scatterer. The reader may refer to [17] and [21] for the detailed discussion on various mathematical properties of the vector basis functions. In fact the surface integral of basis function over adjacent triangles represents moment given by

$$
\begin{aligned}
\iint_{T_{n}^{++}+T_{n}^{-}} \vec{f}_{n} d s & =\frac{l_{n}}{2}\left[\vec{\rho}_{n}^{c+}++\vec{\rho}_{n}^{c-}\right] \\
& =l_{n}\left(\vec{r}_{n}^{c+}-\vec{r}_{n}^{c-}\right)
\end{aligned}
$$

$\vec{\rho}_{n}^{c+}=$ vector between $O_{n}^{+}$and centroid of $T_{n}^{+}$

$$
\vec{\rho}_{n}^{c-}=\text { vector between centroid } T_{n}^{-} \text {and } O_{n}^{-}
$$

and referring to Fig. $4, \vec{r}_{n}^{c+}$ and $\vec{r}_{n}^{c-}$ are the distances to centroids of triangles from the arbitrary reference point.

Referring to the dielectric scatterer shown in Fig. 2, the 


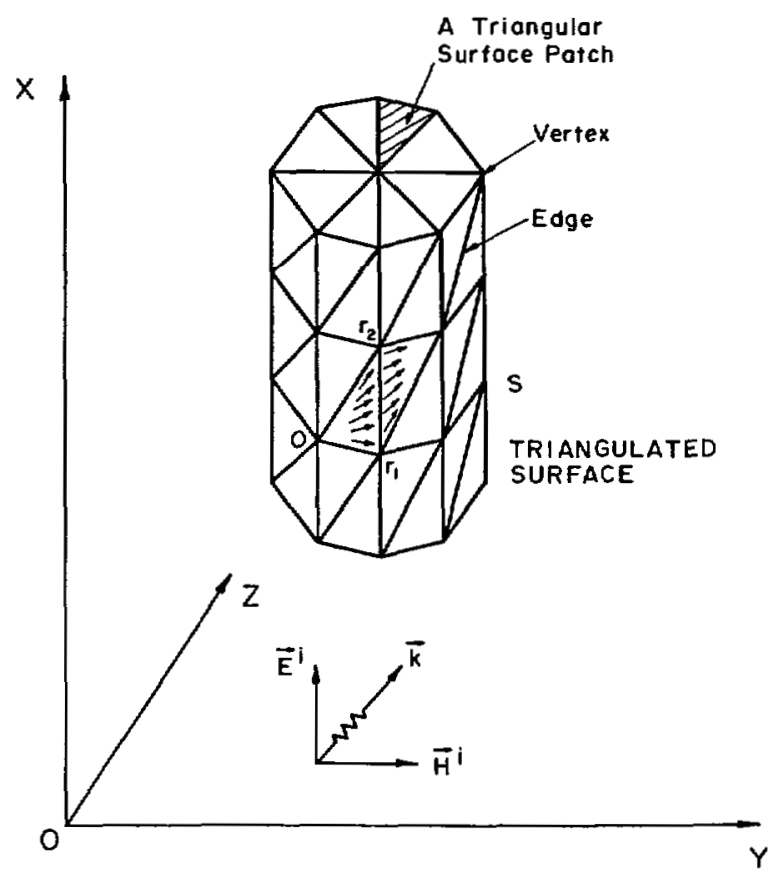

Fig. 2. Finite circular cylinder dielectric scatterer.

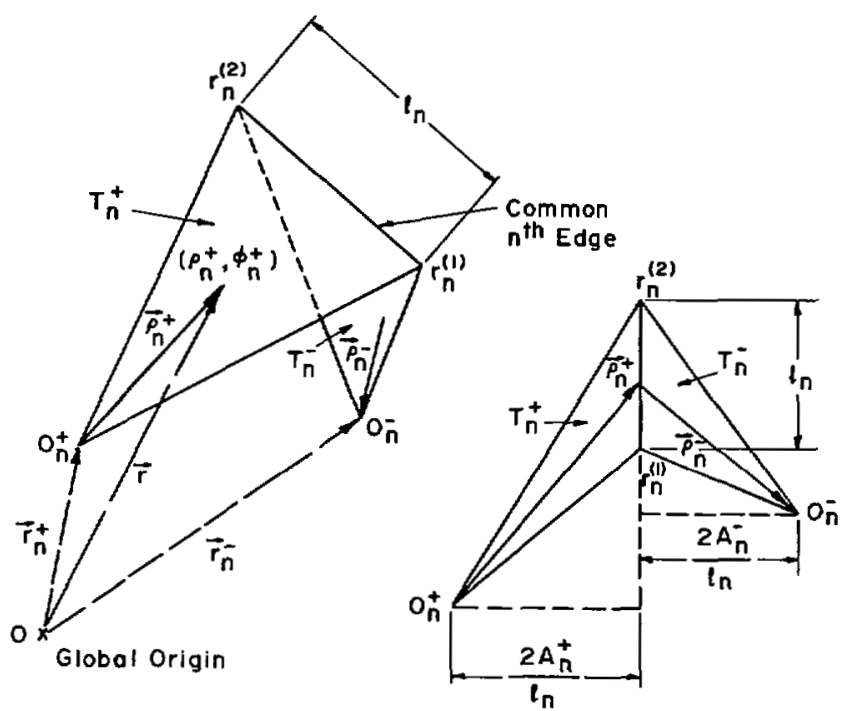

(a)

(b)

Fig. 3. (a) Coordinates of common edge associated with two triangles. (b) Geometry for normal component of basis function at common edge.

surface electric and magnetic current, $\vec{J}$ and $\vec{M}$, distributions are expanded in terms of vector basis functions defined in (9). Let $N$ represents the total number of edges. Then,

$$
\begin{gathered}
\vec{J}\left(\vec{r}^{\prime}\right)=\sum_{n=1}^{N} I_{n} \vec{f}_{n}\left(\vec{r}^{\prime}\right) \\
\vec{M}\left(\vec{r}^{\prime}\right)=\sum_{n=1}^{N} M_{n} \vec{f}_{n}\left(\vec{r}^{\prime}\right)
\end{gathered}
$$

where $I_{n}$ and $M_{n}$ are constants yet to be determined. Since the normal component of $\vec{f}_{n}$ at the $n$th common edge connecting

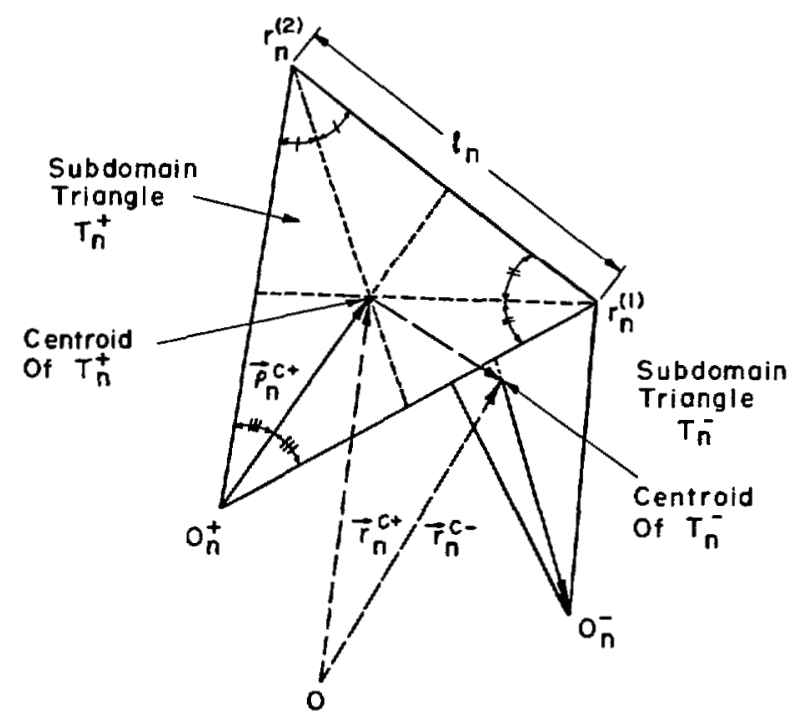

(a)

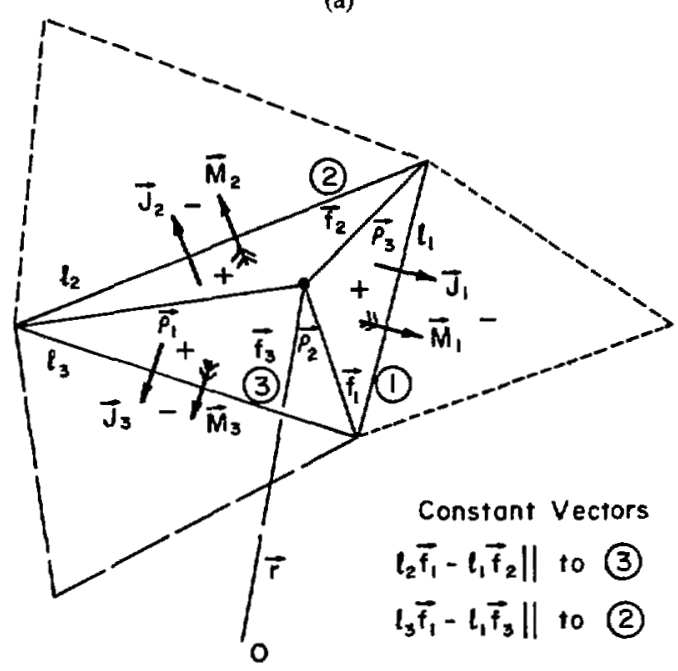

(b)

Fig. 4. (a) Coordinate for calculating centroids and moment of basis vectors. (b) Three edge currents associated with a triangle.

$T_{n}^{+}$and $T_{n}^{-}$is unity, each coefficient of $I_{n}$ and $M_{n}$ can be interpreted as the normal components of the electric and magnetic current density flowing past the $n$th common edge. Further, we note, for a given triangular face, that there are three edges and correspondingly there exists three vector basis functions, Fig. 4. It is also clearly pointed out in basis vectors development [17], [21] that the superposition of the basis functions with a triangle conveniently represent a constant current flowing in an arbitrary direction within the triangle.

\section{Testing of CFIE}

In order to find the current coefficients, the combined field integral equations (8a) and (8b) are tested with respect to testing functions. One suitable choice is to pick testing functions identically same as basis functions, given by (9a)(9c), and test the equations based on the following symmetric product to reduce the operator type integral equations to the corresponding functional type,

$$
\langle\vec{f}, \vec{g}\rangle \equiv \iint_{S} \vec{f} \cdot \vec{g} d S
$$


Hence, testing (8a) and (8b), we obtain

$$
\begin{aligned}
\left\langle\vec{E}^{i}, \vec{f}_{m}\right\rangle= & \left\langle j \omega\left(\vec{A}_{1}+\vec{A}_{2}\right), \vec{f}_{m}\right\rangle+\left\langle\left(\nabla V_{1}+\nabla V_{2}\right), \vec{f}_{m}\right\rangle \\
& +\left\langle\nabla \times\left(\frac{\vec{F}_{1}}{\epsilon_{1}^{\prime}}+\frac{\vec{F}_{2}}{\epsilon_{2}^{\prime}}\right), \vec{f}_{m}\right\rangle, \quad \text { on surface } S
\end{aligned}
$$

and

$$
\begin{aligned}
\left\langle\vec{H}^{i}, \vec{f}_{m}\right\rangle & =\left\langle j \omega\left(\vec{F}_{1}+\vec{F}_{2}\right), \vec{f}_{m}\right\rangle+\left\langle\left(\nabla U_{1}+\nabla U_{2}\right), \vec{f}_{m}\right\rangle \\
& -\left\langle\nabla \times\left(\frac{\vec{A}_{1}}{\mu_{1}}+\frac{\vec{A}_{2}}{\mu_{2}}\right), \vec{f}_{m}\right\rangle, \quad \text { on surface } S
\end{aligned}
$$

where the subscript $m$ denotes an edge formed by two triangles $T_{m}^{+}$and $T_{m}{ }^{-}$. The first-term in (13a) and (13b) can be simplified by evaluating the vector potentials at centroids of respective triangles. Further, the second-term with gradient in (13a) and (13b) can be simplified as

$$
\begin{aligned}
\left\langle\nabla V, \vec{f}_{m}\right\rangle & =-\iint_{S} V\left(\nabla \cdot \vec{f}_{m}\right) d S \\
& =l_{m}\left[\frac{1}{A_{m}^{-}} \iint_{T_{m}^{-}} V d S-\frac{1}{A_{m}^{+}} \int_{T_{m}^{+}} V d S\right] \\
& \simeq l_{m}\left[V\left(r_{m}^{c-}\right)-V\left(r_{m}^{+c}\right)\right] .
\end{aligned}
$$

We note that the integrals in (14b) have been approximated by evaluating the scalar potentials at the respective centroids of the two $T_{m}^{ \pm}$triangles. Similarly, the third-term with "curl" in the above tested equations (13a) and (13b) can be simplified as

$$
\begin{aligned}
\left\langle\nabla \times \vec{A}, \vec{f}_{m}\right\rangle= & \iint_{S}(\nabla \times \vec{A}) \cdot \vec{f}_{m} d S \\
= & \frac{l_{m}}{2 A_{m}^{+}} \iint_{T_{m}^{+}} \vec{\rho}_{m}^{+} \cdot[(\nabla \times \vec{A})]^{+} d S \\
& +\frac{l_{m}}{2 A_{m}^{-}} \iint_{T_{m}^{-}} \vec{\rho}_{m}^{-} \cdot[(\nabla \times \vec{A})]^{-} d s .
\end{aligned}
$$

On substituting the relationships (14) and (15) into the tested form of (13a) and (13b), the following functional form of equations are obtained:

$$
\begin{aligned}
j \omega l_{m} & {\left[\frac{\vec{\rho}_{m}^{c+}}{2} \cdot\left\{\vec{A}_{1}\left(\vec{r}_{m}^{c+}\right)+\vec{A}_{2}\left(\vec{r}_{m}^{c+}\right)\right\}\right.} \\
& \left.+\frac{\vec{\rho}_{m}^{c-}}{2} \cdot\left\{\vec{A}_{1}\left(\vec{r}_{m}^{c-}\right)+\vec{A}_{2}\left(\vec{r}_{m}^{c-}\right)\right\}\right] \\
& +l_{m}\left[\left\{V_{1}\left(\vec{r}_{m}^{c-}\right)-V_{1}\left(\vec{r}_{m}^{c+}\right)\right\}+\left\{V_{2}\left(\vec{r}_{m}^{c-}\right)-V_{2}\left(\vec{r}_{m}^{c+}\right)\right\}\right] \\
& +\left[\left\{\frac{\vec{P}_{1}\left(\vec{r}_{m}^{+}\right)}{\epsilon_{1}^{\prime}}+\frac{\vec{P}_{2}\left(\vec{r}_{m}^{+}\right)}{\epsilon_{2}^{\prime}}\right\}+\left\{\frac{\vec{P}_{1}\left(\vec{r}_{m}^{-}\right)}{\epsilon_{1}^{\prime}}+\frac{\vec{P}_{2}\left(\vec{r}_{m}^{-}\right)}{\epsilon_{2}^{\prime}}\right\}\right] \\
= & l_{m}\left[\frac{\vec{\rho}_{m}^{c+}}{2} \cdot \vec{E}^{i+}\left(\vec{r}_{m}^{c+}\right)+\frac{\vec{\rho}_{m}^{c-}}{2} \cdot \vec{E}^{i-}\left(\vec{r}_{m}^{c-}\right)\right],
\end{aligned}
$$$$
\text { on scatter surface } S, \quad m=1,2,3, \cdots, N \text { edges }
$$

and

$$
\begin{aligned}
j \omega l_{m} & {\left[\frac{\vec{\rho}_{m}^{c+}}{2} \cdot\left\{\vec{F}_{1}\left(\vec{r}_{m}^{c+}\right)+\vec{F}_{2}\left(\vec{r}_{m}^{c+}\right)\right\}\right.} \\
& \left.+\frac{\vec{\rho}_{m}^{c-}}{2} \cdot\left\{\vec{F}_{1}\left(\vec{r}_{m}^{c-}\right)+\vec{F}_{2}\left(\vec{r}_{m}^{c-}\right)\right\}\right] \\
& +l_{m}\left[\left\{U_{1}\left(\vec{r}_{m}^{c-}\right)-U_{1}\left(\vec{r}_{m}^{c+}\right)\right\}+\left\{U_{2}\left(\vec{r}_{m}^{c-}\right)-U_{2}\left(\vec{r}_{m}^{c+}\right)\right\}\right] \\
& -\left[\left\{\frac{\vec{Q}_{1}\left(\vec{r}_{m}^{+}\right)}{\mu_{1}}+\frac{\vec{Q}_{2}\left(\vec{r}_{m}^{+}\right)}{\mu_{2}}\right\}+\left\{\frac{\vec{Q}_{1}\left(\vec{r}_{m}^{-}\right)}{\mu_{1}}+\frac{\vec{Q}_{2}\left(\vec{r}_{m}^{-}\right)}{\mu_{2}}\right\}\right] \\
= & l_{m}\left[\frac{\vec{\rho}_{m}^{c+}}{2} \cdot \vec{H}^{i+}\left(\vec{r}_{m}^{i+}\right)+\frac{\vec{\rho}_{m}^{c-}}{2} \cdot \vec{H}^{i-}\left(\vec{r}_{m}^{c-}\right)\right], \\
& \text { on scatter surface } S, \quad m=1,2,3, \cdots, N \text { edges }
\end{aligned}
$$

where in the above tested equations,

$$
\begin{aligned}
\vec{P}_{1,2}\left(\vec{r}_{m}^{ \pm}\right)=\frac{l_{m}}{2 A_{m}^{ \pm}} \iint_{T_{m}^{ \pm}} \vec{\rho}_{m}^{ \pm} \cdot\left[\nabla \times \vec{F}_{1,2}\left(\vec{r}_{m}^{ \pm}\right)\right] d S \\
\vec{E}^{i+}\left(\vec{r}_{m}^{c+}\right)=\vec{E}^{i}\left(\vec{r}_{m}^{c+}\right) \\
\vec{E}^{i-}\left(\vec{r}_{m}^{c-}\right)=\vec{E}^{i}\left(\vec{r}_{m}^{c-}\right) \\
\vec{Q}_{1,2}\left(\vec{r}_{m}^{ \pm}\right)=\frac{l_{m}}{2 A_{m}^{ \pm}} \iint_{T_{m}^{ \pm}} \vec{\rho}_{m}^{ \pm} \cdot\left[\nabla \times \vec{A}_{1,2}\left(\vec{r}_{m}^{ \pm}\right)\right] d S \\
\vec{H}^{i+}\left(\vec{r}_{m}^{c+}\right)=\vec{H}^{i}\left(\vec{r}_{m}^{c+}\right) \\
\vec{H}^{i-}\left(\vec{r}_{m}^{c-}\right)=\vec{H}^{i}\left(\vec{r}_{m}^{c-}\right) .
\end{aligned}
$$

In the above functional equations (16a) and (16b), the vector and the scalar potentials $\vec{A}_{i}, \vec{F}_{i}, V_{i}, U_{i}$ are given by (3a)-(3d). The $\vec{P}_{i}$ and $\vec{Q}_{i}$ terms containing the curl operations can $\overrightarrow{b e}$ further simplified as, for $i=1,2$ regions,

$$
\begin{aligned}
& {\left[\nabla \times \vec{F}_{i}(\vec{r})\right]=\frac{\epsilon_{i}^{\prime}}{4 \pi} \int_{S} \vec{M}\left(\vec{r}^{\prime}\right) \times \nabla^{\prime} G_{i}\left(\vec{r}, \vec{r}^{\prime}\right) d S\left(\vec{r}^{\prime}\right)} \\
& {\left[\nabla \times \vec{A}_{i}(\vec{r})\right]=\frac{\mu_{i}}{4 \pi} \int_{S} \vec{J}\left(\vec{r}^{\prime}\right) \times \nabla^{\prime} G_{i}\left(\vec{r}, \vec{r}^{\prime}\right) d S\left(\vec{r}^{\prime}\right)}
\end{aligned}
$$

where the symbol $f f$ represents Cauchy principle value of the integral. In the numerical development, the integrals defined in (17a) and (18a) will be evaluated numerically [17], [21] using seven point integration method principally applicable to triangular distributions.

\section{Matrix Equation (CFIE)}

The electric current and the magnetic current expansion terms defined in (11a) and (11b) are now substituted into the CFIE tested (16a) and (16b) to reduce the functional form of the equation to a corresponding partitioned matrix equation [17], 
[21]:

$$
\left[\begin{array}{ll}
{\left[Z_{m n}^{J J}\right]} & {\left[C_{m n}^{J M}\right]} \\
{\left[D_{m n}^{M J}\right]} & {\left[Y_{m n}^{M M}\right]}
\end{array}\right]\left[\begin{array}{c}
{\left[I_{n}\right]} \\
{\left[M_{n}\right]}
\end{array}\right]=\left[\begin{array}{l}
{\left[V_{m}\right]} \\
{\left[H_{m}\right]}
\end{array}\right]
$$

where the various matrix elements are given by the following, for $m=1,2,3, \cdots, N$ edges and $n=1,2,3, \cdots, N$ edges.

Elements of diagonal submatrix for electric current:

$$
\begin{array}{r}
Z_{m n}^{J J}=l_{m}\left[\frac{\vec{\rho}_{m}^{c+}}{2} \cdot \sum_{i=1}^{2}\left(j k_{i} \eta_{i}\right) \vec{A}_{i_{m n}}^{+}+\frac{\vec{\rho}_{m}^{c-}}{2} \cdot \sum_{i=1}^{2}\left(j k_{i} \eta_{i}\right) \vec{A}_{i_{m n}}^{-}\right. \\
\left.+\sum_{i=1}^{2}\left(\frac{-\eta_{i}}{j k_{i}}\right)\left\{\Phi_{i_{m n}}^{-}-\Phi_{i_{m n}}^{+}\right\}\right] \cdot
\end{array}
$$

Elements of diagonal submatrix for magnetic current:

$$
\begin{aligned}
Y_{m n}^{M M}=l_{m}\left[\frac{\vec{\rho}_{m}^{c+}}{2} \cdot\right. & \sum_{i=1}^{2}\left(\frac{j k_{i}}{\eta_{i}}\right) \vec{F}_{i_{m n}}^{+}+\frac{\vec{\rho}_{m}^{c-}}{2} \cdot \sum_{i=1}^{2}\left(\frac{j k_{i}}{\eta_{i}}\right) \vec{F}_{i_{m n}}^{-} \\
& \left.+\sum_{i=1}^{2}\left(\frac{-1}{j k_{i} \eta_{i}}\right)\left\{\Psi_{i_{m n}}^{-}-\Psi_{i_{m n}}^{+}\right\}\right] \cdot
\end{aligned}
$$

Elements of off-diagonal submatrix:

$$
\begin{aligned}
C_{m n}^{J M} & =\left[\sum_{i=1}^{2} P_{i_{m n}}^{+}+\sum_{i=1}^{2} P_{i_{m n}}^{-}\right] . \\
D_{m n}^{m J} & =\left[\sum_{i=1}^{2} Q_{i_{m n}}^{+}+\sum_{i=1}^{2} Q_{i_{m n}^{-}}^{-}\right] .
\end{aligned}
$$

Elements of electric and magnetic field excitation:

$$
\begin{aligned}
& V_{m}=l_{m}\left[\frac{\vec{\rho}_{m}^{c+}}{2} \cdot \vec{E}^{i+}\left(\vec{r}_{m}^{c+}\right)+\frac{\vec{\rho}_{m}^{c-}}{2} \cdot \vec{E}^{i-}\left(\vec{r}_{m}^{c-}\right)\right] \\
& H_{m}=l_{m}\left[\frac{\vec{\rho}_{m}^{c+}}{2} \cdot \vec{H}^{i+}\left(\vec{r}_{m}^{c+}\right)+\frac{\vec{\rho}_{m}^{c-}}{2} \cdot \vec{H}^{i-}\left(\vec{r}_{m}^{c-}\right)\right]
\end{aligned}
$$

and, the vector and the scalar potential integrals take the following form [17], [21], for $i=1,2$ :

$$
\begin{aligned}
\vec{A}_{i_{m n}}^{ \pm} & =\frac{1}{4 \pi} \iiint_{\left(T_{n}^{+}+T_{n}^{-}\right)} \vec{f}_{n}\left(\vec{r}^{\prime}\right) G_{i}\left(\vec{r}_{m}^{c \pm}, \vec{r}^{\prime}\right) d S\left(\vec{r}^{\prime}\right) \\
& =\vec{F}_{i_{m n}^{ \pm}}^{ \pm} \\
\Phi_{i_{m n}}^{ \pm} & =\frac{1}{4 \pi} \iiint_{\left(T_{n}^{+}+T_{n}^{-}\right)}\left[\nabla_{S}^{\prime} \cdot \vec{f}_{n}\left(\vec{r}^{\prime}\right)\right] G_{i}\left(\vec{r}_{m}^{c \pm}, \vec{r}^{\prime}\right) d S\left(\vec{r}^{\prime}\right) \\
& =\Psi_{i_{m n}}^{ \pm} \\
P_{i_{m n}}^{ \pm}= & \frac{l_{m}}{2 A_{m}^{ \pm}} \int_{T_{m}^{ \pm}} \vec{\rho}_{m}^{ \pm} \cdot\left[\frac{1}{4 \pi} \iiint_{\left(T_{n}^{+}+T_{n}^{-}\right)} \vec{f}_{n}\right. \\
& \left.\times \nabla^{\prime} G_{i}\left(\vec{r}_{m}^{ \pm}, \vec{r}^{\prime}\right) d S\left(\vec{r}^{\prime}\right)\right] d S(\vec{r}) \\
= & Q_{i_{m n}}^{ \pm}
\end{aligned}
$$

and

$$
\begin{gathered}
G_{i}\left(\vec{r}_{m}^{ \pm}, \vec{r}^{\prime}\right)=\frac{e^{-j k} i^{R^{ \pm}}}{R^{ \pm}} \\
R^{ \pm}=\left|\vec{r}_{m}^{ \pm}-\vec{r}^{\prime}\right| \\
\nabla^{\prime} G_{i}\left(\vec{r}_{m}^{ \pm}, \vec{r}^{\prime}\right)=\left(\vec{r}_{m}^{ \pm}-\vec{r}^{\prime}\right)\left(1+j k_{i} R^{ \pm}\right) \frac{e^{-j k_{i} R^{ \pm}}}{\left(R^{ \pm}\right)^{3}} .
\end{gathered}
$$

\section{EfFicient Numerical Algorithm DeVelopment}

The integrals (22a), (22c) and (23a) are in a convenient form for numerical evaluation. The simplification of these integrals are discussed in detail in [21], which are useful for numerical algorithm development. The matrix equation (20) can be inverted to obtain the electric and magnetic current coefficients, $I_{n}$ and $M_{n}$.

We note that the various matrix elements can be easily generated by considering faces rather than edges. This cuts down by approximately ninefold, computer time required to generate matrix elements [21]. We further note that the matrix elements $Z_{m n}$ and $Y_{m n}$ are similar except for floating constants which can be conveniently incorporated while filling matrix elements; so that one has to generate only $Z_{m n}$ matrix elements and the $Y_{m n}$ elements are obtained by changing multiplying constants while filling in matrix elements. Similarly $C_{m n}$ and $D_{m n}$ matrix elements differ by just a multiplying constant; so that one is to generate just $C_{m n}$ elements only and the $D_{m n}$ elements are obtained directly from the $C_{m n}$ elements. Also referring to the expression (21), the elements of the submatrices contain terms belonging to both regions one and two. The various potential integral expressions for the regions 1 and 2 are in fact identical except for the electrical characteristics which appear in the propagation constants and in the multiplication constants. For an efficient numerical algorithm development and to save computer time, same subroutines are simultaneously utilized for calculating region 1 and region 2 integral terms which principally make up various matrix elements.

To obtain the electric and the magnetic current distributions one can either directly invert the matrix equation (20), or the matrix equation (20) can be rearranged so as to eliminate one unknown, and resubstituted back to obtain the second unknown. This takes less computer time than directly inverting a large composite matrix equation. Another possible method seems to be application of the iterative methods [10]. Detail studies are still underway in this specific area to utilize iterative schemes.

\section{Numerical Results-Homogeneous Dielectric Sphere and Fintte Circular Cylinder}

To demonstrate applicability of the above formulation and to validate computer algorithms, numerical results are presented for the case of a homogeneous dielectric sphere and for the case of a homogeneous dielectric finite circular cylinder located in free space and excited by a plane wave.

The surface of the sphere is first modeled in terms of triangles having arbitrary edges and vertices arranged to depict the shape of a sphere. Fig. 5(a) shows the plan view (top 


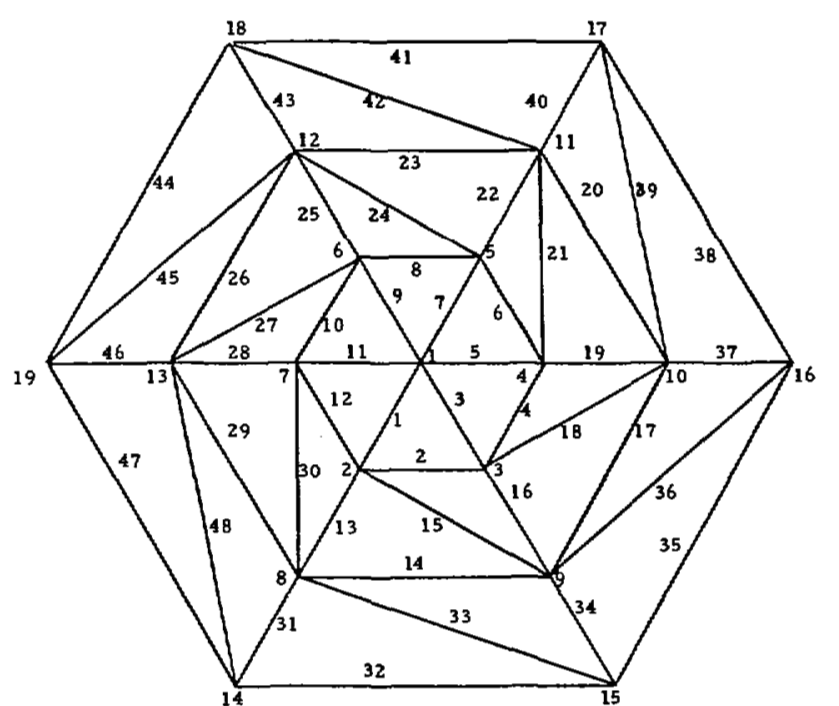

(a)

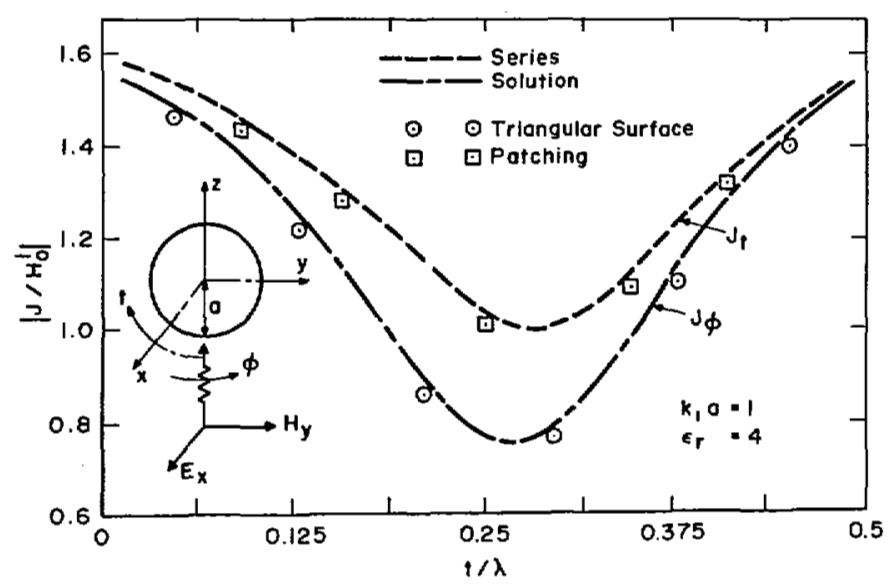

(b)

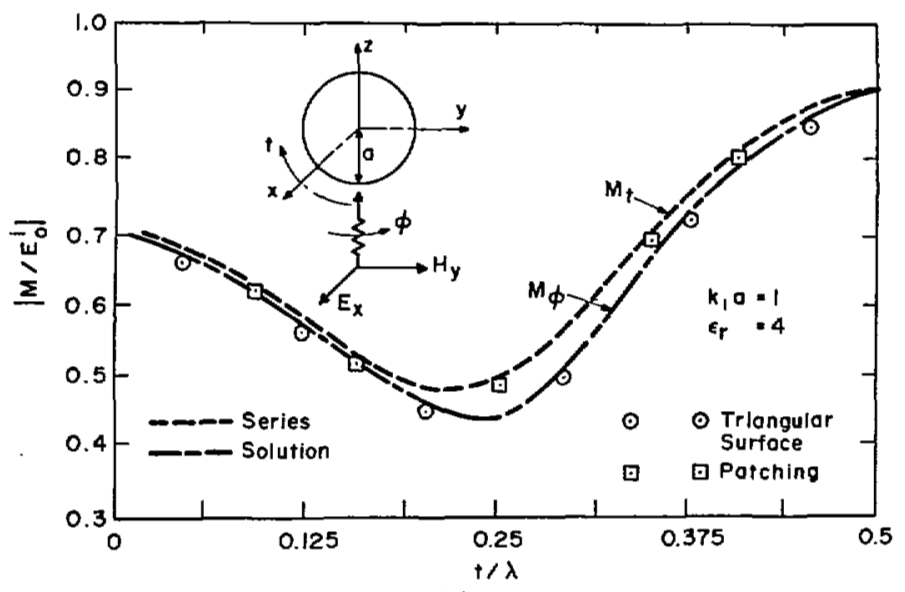

(c)

Fig. 5. (a) Triangular surface patching for top half of sphere (plan view). (b) Equivalent surface electric current distribution on a homogeneous dielectric sphere. (c) Equivalent surface magnetic current distribution on a homogeneous dielectric sphere.

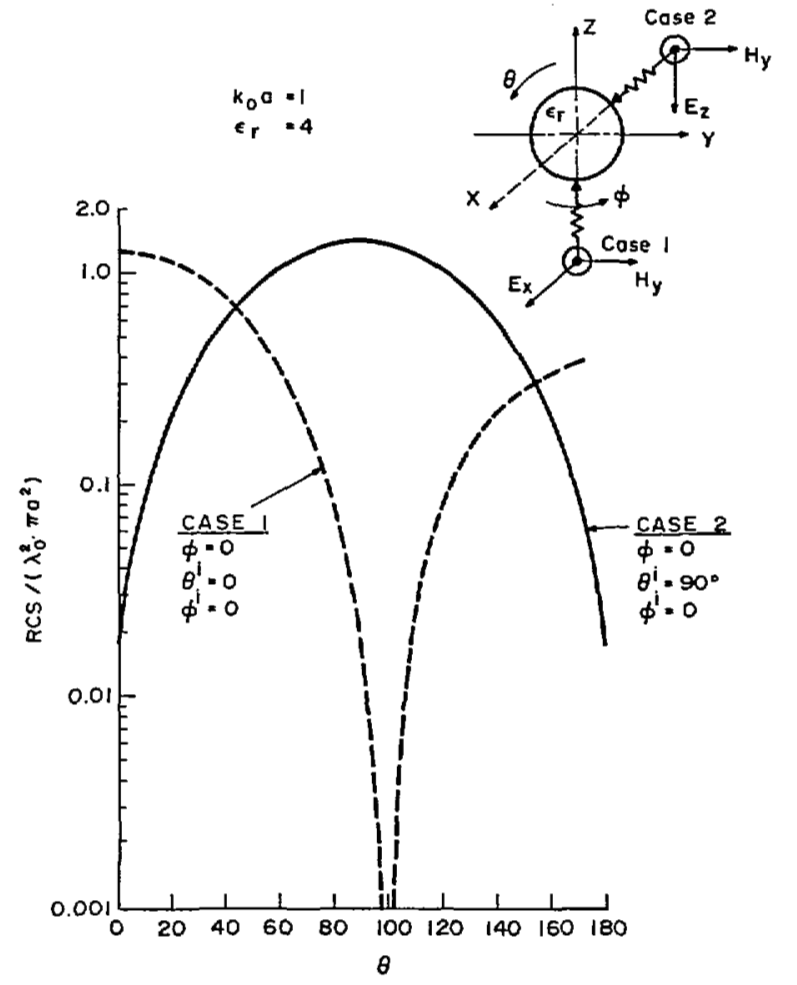

Fig. 6. Bistatic radar cross section of homogeneous dielectric sphere.

hemisphere only) of the triangular scheme adopted. There are in total 60 triangular faces consisting of 90 edges at which the unknown normal components of the electric and magnetic currents solved. The matrix size adopted to check the accuracy is $180 \times 180$. The electrical size of the sphere is $k_{1} a=1$ where the free space propagation constant $k_{1}=2 \pi / \lambda_{0}$ and the radius of the sphere is $a$. The relative dielectric constant of the sphere is $\epsilon_{r}=4$. The sphere is located in free space and is excited by an axial incident plane wave. In Figs. 5(b) and 5(c) are shown the induced electric and the induced magnetic currents on the surface of the sphere along a circumferential arc in $x z$ plane. Along the arc, there are two components of the electric currents $J_{t}$ and $J_{\phi}$; and magnetic currents $M_{t}$ and $M_{\phi}$. The results of the induced electric currents are shown normalized with respect incident magnetic field and similarly the induced magnetic currents are shown normalized with respect to incident electric field. The induced surface fields for a sphere problem can also be obtained by the eigenfunction analysis using spherical harmonic functions. The results of this approach are also shown in Figs. 5(b) and 5(c). The CFIE/ MM solution based on triangular surface patching has good agreement with the eigenfunction series solution [2].

Fig. 6 gives the computed bistatic radar cross section for the homogeneous dielectric sphere based on the equivalent surface electric and magnetic currents. The radar cross section results are shown as a function of $\theta$ in the vertical plane cut $\phi=0$ for two different angles of incidence; one along axial excitation and the other along broad excitation. The results check very well with the results based on body of revolution treatment [5].

Figs. 7(a) and 7(b) show the distribution of surface electric and magnetic currents for the case of homogeneous dielectric 


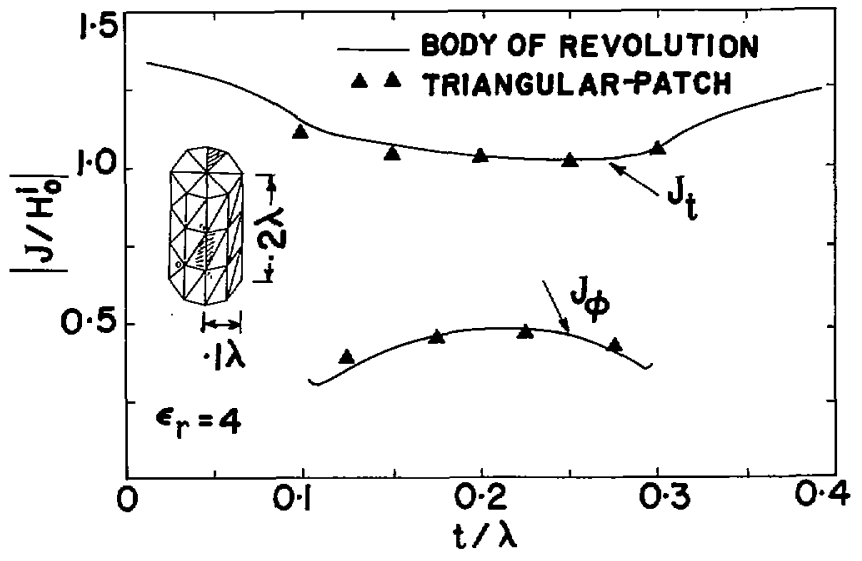

(a)

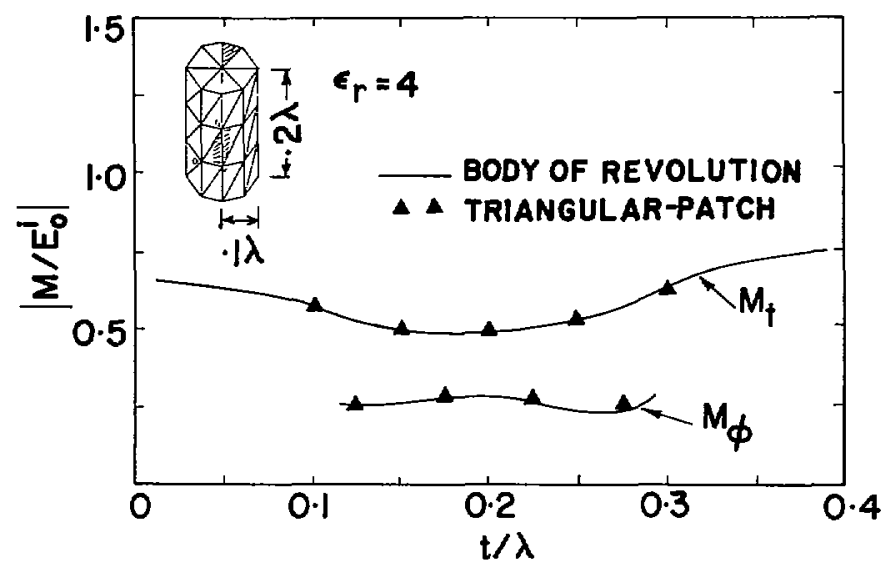

(b)

Fig. 7. (a) Electric surface current distribution on a dielectric finite circular cylinder due to axially incident plane wave. (b) Magnetic surface current distribution on a dielectric finite circular cylinder due to axially incident plane wave.

finite circular cylinder located in free space and externally excited by a plane wave. The results of the surface induced currents agree very well with those obtained based on the body of revolution [19] treatment.

\section{CONCLUSION}

Based on the method of moments technique and the combined field integral equations, this paper reported recent developments and extensions to analyze electromagnetic scattering by arbitrary shaped three dimensional homogeneous lossy and lossless objects. Similar to the case of conducting objects, the arbitrary geometrical shapes have been modeled using surface triangular patches. Efficient and simple numerical algorithms are also developed; and validations reported here for two canonical dielectric scatterers namely a sphere and a finite circular cylinder. The numerical technique discussed here can be conveniently extended to analyze electromagnetic scattering by objects which are homogeneous, lossy, but having diagonizable tensor material characteristics [17]. The results of this study and their verifications will be reported in an upcoming paper.

\section{REFERENCES}

[1] J. J. Bowman, T. B. A. Senior, and P. L. E. Uslenghi, Electromagnetic and Acoustic Scattering by Simple Shapes. Amsterdam:
North-Holland, 1969.

[2] R. W. P. King and C. W. Harrison, "Scattering by imperfectly conducting spheres," AFCRL-70-0483, Cruft Lab. Rep. by Harvard Univ., 1970.

[3] M. G. Andreasen, "Scattering from bodies of revolution," IEEE Trans. Antennas Propagat., vol. AP-13, pp. 303-310, Mar. 1965.

[4] V. A. Erma, "Exact solution for the scattering of electromagnetic waves from bodies of arbitrary shape, III, Obstacles with arbitrary electromagnetic properties," Phys. Rev., vol. 179, pp. 1238-1246, Mar. 1969.

[5] T. K. Wu, "Electromagnetic scattering from arbitrarily-shaped lossy dielectric bodies," Ph.D. dissertation, Univ. Mississippi, University, MS, May 1976.

[6] C. Yeh, "Perturbation approach to the diffraction of electromagnetic waves by arbitrarily shaped dielectric obstacles," Phys. Rev., vol. 135, no. 5A, pp. A1193-A1201, Aug. 1964.

[7] J. H. Richmond, "Scattering by dielectric cylinder of arbitrary cross section shape," IEEE Trans. Antennas Propagat., vol. AP-13, pp. 334-341, May 1965.

[8] D. H. Schaubert, D. R. Wilton, and A. W. Glisson, "A tetrahedral modeling for electromagnetic scattering by arbitrary shaped homogeneous dielectric bodies," IEEE Trans. Antennas Propagat., vol. AP32, no. 1 , pp. 77-85, Jan. 1984.

[9] D. T. Borup and O. P. Gandhi, "Fast-Fourier transform method for calculation of SAR distributions in finely discretized inhomogeneous models of biological bodies," IEEE Trans. Microwave Theory Tech., vol. MTT-32, no. 4, pp. 355-360, Apr. 1984.

[10] T. K. Sarkar and S. M. Rao, "An iterative method for solving electrostatic problems," IEEE Trans. Antennas Propagat., vol. AP. 30, pp. 611-616, July 1982.

[11] D. E. Livesay and K. M. Chen, "Electromagnetic fields induced inside arbitrarily shaped biological bodies," IEEE Trans. Microwave Theory Tech., vol. MTT-22, no. 12, pp. 1273-1280, Dec. 1974.

[12] P. C. Waterman, "Matrix formulation of electromagnetic scattering," Proc. IEEE, vol. 53, no. 8, pp. 805-812, Aug. 1965.

[13] S. Govind, "Numerical computation of electromagnetic scattering by inhomogeneous penetrable bodies," Ph.D. dissertation, Univ. Mississippi, University, MS, Dec. 1978.

[14] K. K. Mei, "Unimoment method of solving antenna and scattering problems," IEEE Trans. Antennas Propagat., vol. AP-22, pp. 760766, Nov. 1974.

[15] P. C. Waterman, "Scattering by dielectric obstacles," Alta. Freq. vol. 38, (Speciale), p. 348, 1969.

[16] S. Strom, "T-Matrix for electromagnetic scattering from an arbitrary number of scatterers with continuously varying electromagnetic properties," Phys. Rev. D, vol. 10, pp. 2685-2690, Oct. 1974.

[17] K. R. Umashankar and A. Taflove, "Analytical models for electromagnetic scattering," Part-I, Final Rep., Contract F19628-82-C-0140 to RADC/ESD, Hanscom Air Force Base, MA, June 1984.

[18] R. J. Pogorzelski, "On the numerical computation of scattering from inhomogeneous penetrable objects," IEEE Trans. Antennas Propagat., vol. AP-26, pp. 616-618, July 1978.

[19] A. W. Glisson, "On the development of numerical techniques for treating arbitrarily-shaped surfaces," Ph.D. dissertation, Univ. Mississippi, University, MS, 1978.

[20] S. M, Rao, D. R. Wilton, and A. W. Glisson, "Electromagnetic scattering by surfaces of arbitrary shape," IEEE Trans. Antennas Propagat., vol. AP-30, pp. 409-418, May 1982.

[21] S. M. Rao, "Electromagnetic scattering and radiation of arbitrarilyshaped surfaces by triangular patch modeling, "Ph.D. dissertation, Univ. Mississippi, University, MS, Aug. 1980.

[22] J. R. Mautz and R. F. Harrington, "Electromagnetic scattering from a homogeneous material body of revolution," Arch. Elek. Ubertragung, vol. 33, no. 4, pp. 71-80, Apr. 1979.

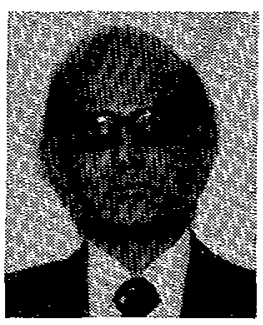

Korada Umashankar (S'69-M'75-SM'81) received the B.E. degree from Mysore University, India, in 1962, the M.E. degree from the Indian Institute of Science, Bangalore, India, in 1964, and the Ph.D. degree from University of Mississippi, University, MS, in 1974, all in electrical engineering.

From 1964-1969, he was Assistant Professor and Head of the Department of Electrical Engineering, College of Engineering, Karnatak University, Hubli, India. During 1974-1975 he was a Postdoc- 
toral Research Associate, and during 1975-1977 he was Assistant Professor of Electrical Engineering, University of Mississippi. From 1977-1979, he was the National Research Council Visiting Fellow at the Airforce Weapons Laboratory, Kirtland AFB, NM, involved with the simulator studies and electromagnetic transients. During 1979-1984 he was Senior Engineer in the Electronics Division, IIT Research Institute, Chicago, IL, involved with the development of numerical models for electromagnetic interaction with complex objects. Currently he is Associate Professor of Electrical Engineering, University of Illinois at Chicago, Chicago, IL. His primary research work has been in the development of analytical and mathematical techniques in electromagnetic theory, EMP/EMC interaction, and EM simulation studies.

Dr. Umashankar is a member of Sigma Xi, Eta Kappa Nu, AAAS, and Commission B-URSI.

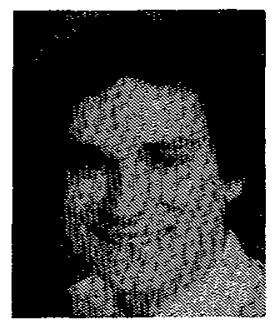

Allen Taflove (M'75-SM'84) was born in Chicago, IL, on June 14, 1949. He received the B.S., M.S., and Ph.D. degrees in electrical engineering from Northwestern University, Evanston, IL, in 1971,1972 , and 1975 , respectively.

From 1975 to 1984 , he was a staff member at IIT Research Institute in Chicago, II, holding the positions of Associate Engineer, Research Engineer, and Senior Engineer. There, his research was concerned with electromagnetic wave penetration and scattering, power-frequency coupling of earth- return transmission systems, and development of novel techniques for recovery of oil from oil shale, tar sand, and conventional wells based upon in situ radio-frequency heating. He was principal investigator on eight externally funded programs in these areas, including five programs which contributed to the early development of the finite difference-time domain numerical modeling approach for complex electromagnetic wave interaction problems. In 1984, he joined the Electrical Engineering Department at Northwestern University, Evanston, IL, as an Associate Professor. Since then, he has developed several research programs in the area of computational electromagnetics. His current interests include applications of recent super-computers, wave penetration and scattering by large, complex structures, inverse scattering/target synthesis, and the new on-surface radiation condition theory.

Dr. Taflove is a member of Et Kappa Nu, Tau Beta Pi, Sigma Xi, AAAS, and the New York Academy of Sciences. He has been granted eight patents for his in situ oil recovery inventions, and was senior author of a paper awarded the Best Paper prize at the IEEE 1983 International Symposium on Electromagnetic Compatibility, Washington, DC.

Sadasiva M. Rao, for a photograph and biography please see page 418 of the May 1982 issue of this TRANSACTIONS. 